\title{
Exclude ECG Data From Statistics Indicator
}

National Cancer Institute

\section{Source}

National Cancer Institute. Exclude ECG Data From Statistics Indicator. NCI Thesaurus.

Code C119824.

Specifies whether the ECG result values should be excluded from the statistical analysis. 